\title{
TRIM27 promotes the development of esophagus cancer via regulating PTEN/AKT signaling pathway
}

\author{
Liang Ma ${ }^{1,2}$, Ninghua Yao ${ }^{3}$, Ping Chen ${ }^{2^{*}}$ and Zhixiang Zhuang ${ }^{1 *}$ (D)
}

\begin{abstract}
Background: Tripartite motif-containing 27 (TRIM27) belongs to the TRIM protein family, which is closely related to the progression of some certain human cancers. Nevertheless, the biological function of TRIM27 in esophageal squamous cell carcinoma (ESCC) is still not clear. The aim of present research is to examine the function of TRIM27 in ESCC cells.

Methods: In the present study, RNA interference (RNAi) and lentiviral vector were used to knockdown and overexpression of TRIM27 in ESCC cells respectively. qRT-PCR and western blot were used to examine the expression of TRIM27 in ESCC cells. Cell counting kit-8 (CCK-8) assay was performed to determine the proliferation of cells.

Results: Our analyses indicated that TRIM27 was a pro-proliferation factor in ESCC cells. Moreover, overexpression of TRIM27 deeply suppressed the apoptosis of ESCC cells and accelerated its glucose uptake. In addition, an AKT inhibitor LY294002 was used to determine the connection between TRIM27 and AKT in ESCC cells. Our results demonstrated that TRIM27 has involved in the PI3/AKT signaling pathway. Moreover, TRIM27 interacted with PTEN and mediated its poly-ubiquitination in ESCC cells. Importantly, the glycolysis inhibitor 3-BrPA also inhibited the effect of TRIM27 on ESCC cells. Hence, TRIM27 also participated in the regulation of energy metabolism in ESCC cells.
\end{abstract}

Conclusions: This research not only gained a deep insight into the biological function of TRIM27 but also elucidated its potential target and signaling pathway in human ESCC cells.

Keywords: Esophagus cancer, TRIM27, PI3/AKT, PTEN

\section{Background}

Esophageal squamous cell carcinoma (ESCC) is one of the common death related cancers worldwide, which ranks the sixth place in cancer mortality [1]. Although the traditional treatments (radiation, chemotherapy or esophagogastric resection) contribute to the treatment of ESCC patients, the overall 5-year survival rate is still less than 20\% [2]. Previous report has demonstrated that

\footnotetext{
*Correspondence: doc_pchen@126.com; zzx22066@163.com

1 Department of Oncology, The Second Affiliated Hospital of Soochow University, Sanxiang Road No. 1055, Gusu District, Suzhou 215004, Jiangsu, China

${ }^{2}$ Department of Oncology, First People's Hospital of Yancheng, The Forth Affiliated Hospital of Nantong University, Yulong West Road No.166,

Tinghu District, Yancheng 224001, Jiangsu, China

Full list of author information is available at the end of the article
}

ESCC can be possibly cured at its early phase [3]. Therefore, the effective biomarkers for diagnosing ESCC are urgently needed.

Tripartite motif-containing 27 (TRIM27) is one of the family members of TRIM, which inherits the basic structure of TRIM family [4]. As a DNA binding protein, TRIM27 consists of a RING finger protein domain, a B-box type I domain, and a B-box type II domain and exhibits a RBCC motif at the N-terminus [5]. Previous report has demonstrated that TRIM27 accelerates the progression of colorectal cancer [5]. Moreover, it have been confirmed that inhibiting the expression of TRIM27 suppresses the proliferation of ovarian and nasopharyngeal cancer cells respectively $[6,7]$. In addition, TRIM27 positively regulates the TNF- $\alpha$-induced apoptosis [8]. Importantly, some single nucleotide polymorphisms 
(SNPs) of TRIM27 is associated with human ESCC [9]. However, the underlying molecule network of TRIM27 is still less identified in human ESCC.

PI3K/AKT signaling pathway plays a key role in the progression of human cancers, which is identified as a promising target for anti-cancer therapy [10]. Previous reports have confirmed that TRIM59 and TRIM27 promote the proliferation of CRC cells through regulating PI3K/AKT signaling pathway [5, 11]. Moreover, TRIM14 promotes the activity of PI3K/AKT signaling pathway in osteosarcoma cells [12]. Further, suppressing the PI3K/ AKT signaling pathway contributes to inhibiting the proliferation of ESCC cells [13, 14]. However, the detailed relationship between TRIM27 and PI3/AKT pathway remains unclear in human ESCC cells.

The aim of present study is to explore the biological function of TRIM27 in ESCC cells. RNA interference (RNAi) and lentiviral vector were used to silencing and overexpression of TRIM27 in human ESCC cells. Our research not only provided novel evidences to understand the biological function of TRIM27 in human ESCC cells but also elucidated its potential target and signaling pathway in ESCC cells.

\section{Materials and methods}

\section{ESCC tissue specimens}

Esophageal squamous cell carcinoma tissues $(n=25)$ and adjacent-matched noncancerous samples $(n=18)$ were collected from The Second Affiliated Hospital of Soochow University, Suzhou, Jiangsu, China. All tissues were snap-frozen in liquid nitrogen, stored at $-80{ }^{\circ} \mathrm{C}$.

\section{Cell culture}

The cell lines used in this research were TE-1, TE-11, ECA-109, KYSE150 and HEEC, which were purchased from Shanghai biology institute (Shanghai, China). 10\% fetal bovine serum (GIBCO, USA) was added to culture media that containing $2 \mathrm{mM} \mathrm{L}$-glutamine and $1 \%$ penicillin/streptomycin (Solarbio, China). Cells were grown in DMEM Medium (Trueline, USA) and maintained in a $5 \% \mathrm{CO}_{2}$, at $37{ }^{\circ} \mathrm{C}$ incubator. This study was in agreement with the Declaration of Helsinki. The AKT inhibitor LY294002 $(25 \mu \mathrm{mol} / \mathrm{L}, \mathrm{S} 1105$, Selleck, USA) and glycolytic inhibitor 3-BrPA (20 $\mu \mathrm{mol} / \mathrm{L}, 1113-59-3$, Sigma, USA) were dissolved in DMSO (D2650, Sigma, USA) and used to culture cells.

\section{RNA isolation and real-time PCR}

Total RNA from different samples were isolated by using TRIzol Reagent (Invitrogen, USA). Then, cDNA synthesis kit (Fermentas, Canada) was used to reverse transcribe RNA into complementary DNA (cDNA). The program of the real-time PCR reaction was listed as follows: $95^{\circ} \mathrm{C}$ for
$10 \mathrm{~min}$ followed by 40 cycles of $95^{\circ} \mathrm{C}$ for $15 \mathrm{~s}$ and $60{ }^{\circ} \mathrm{C}$ for $45 \mathrm{~s}$. GAPDH was used to normalize the gene expression. $2^{-\Delta \Delta C t}$ method was applied to calculate the relative gene expression. All data represented the mean of three replicates. Primer sequences are provided in Additional file 1.

\section{Knockdown and overexpression of TRIM27}

For silencing human TRIM27 (NM_006510.4), three shot interference RNAs (siRNA) that targeting TRIM27 were synthesized (Major, Shanghai, China) and subsequently transfected into the KYSE150 and TE-11 cells respectively by using Lipofectamine 2000 (Invitrogen, USA). Meanwhile, a nonspecific scrambled siRNA sequence was transfected into KYSE150 and TE-11 cells as negative control (siNC). The targeting locus and sequence of TRIM27 siRNA is provided in Additional file 1: Table S1.

As for overexpression of TRIM27, a lentiviral plasmid (pLVX-puro) containing the full-length human TRIM27 cDNA sequence and a mock plasmid (oeNC) were transiently transfected into TE-1 cells respectively.

\section{Western blot}

RIPA lysis buffer (JRDUN, Shanghai, China) was used to extract protein as indicated. An enhanced BCA protein assay kit (Thermo Fisher, USA) was utilized to estimate the protein content. Total protein $(25 \mu \mathrm{g})$ was fractionated by using $10 \%$ SDS-PAGE and transferred to a nitrocellulose membrane (Millipore, USA) for $2 \mathrm{~h}$, which were probed at $4{ }^{\circ} \mathrm{C}$ for $12 \mathrm{~h}$ with the primary antibodies followed by incubation for $1 \mathrm{~h}$ at $37^{\circ} \mathrm{C}$ with the secondary antibody (HRP-labeled goat anti rabbit IgG antibody; 1:1000, Beyotime, China). An enhanced chemiluminescence system (Tanon, China) was utilized to quantify the protein content. Each analysis was detected in triplicate. GAPDH was used as the internal reference. The crucial information of the primary antibodies was listed in Additional file 1: Table S2.

\section{Cell proliferation assay}

Cell counting kit-8 (CCK-8) assay kits (SAB, USA) was used to examine cell proliferation. All procedures were performed according to the instruction of the manufacture. In brief, cells were seeded in 96-well plates and incubated with CCK-8 solution (1:10) for $1 \mathrm{~h}$. Then, OD450 value was examined by microplate reader (Pulangxin, China) at 12, 24 and $48 \mathrm{~h}$ after seeding. The experiment was independently repeated thrice at each time point.

\section{Cell apoptosis}

Briefly, Annexin V-fluorescein isothiocyanate (FITC) apoptosis detection kit (Beyotime, China) was used to examine the apoptosis rate of cells according to the 
instructions of the manufacturer. After $48 \mathrm{~h}$ of viral infection, flow cytometer (BD, USA) were utilized to determine cells. Three replications were needed for each sample.

\section{Glucose transport}

In brief, the glucose analog 2-NBDG kits (Biovision, San Francisco, USA) were used as a fluorescent probe for determining the activity of Glucose transport. In order to examine the uptake of 2-NBDG, a total of $5 \times 10^{5}$ cells from different groups were seeded in 6 -well plates. Then, all cells were pre-incubated in Krebs-Ringer bicarbonate (KRB) buffer (glucose free) for 15 min after maintaining in a $5 \% \mathrm{CO}_{2}$ atmosphere at $37^{\circ} \mathrm{C}$ for $24 \mathrm{~h}$. After that, cells were incubated in fresh KRB buffer supplemented with 2-NBDG for $45 \mathrm{~min}$ at $37^{\circ} \mathrm{C}, 5 \% \mathrm{CO}_{2}$. Flow cytometry using a GloMax ${ }^{\circledR}$-Multi + flow cytometer (Promega, USA) was used to quantitatively analyze the stained cells.

\section{Co-immunoprecipitation (Co-IP)}

For IP, whole-cell extracts were prepared after transfection or stimulation with appropriate ligands, followed by incubation overnight with the appropriate antibodies plus Protein A/G beads (Santa Cruz Biotechnology, USA). Beads were washed five times and separated by SDS-PAGE. Western blot was performed by using the antibodies as indicated above.

\section{Ubiquitination assay}

KYSE150 cells were transfected with siNC or siTRIM27, the cells were lysed in 1\% SDS-containing radio immunoprecipitation assay (RIPA) buffer by sonication on ice. Then, Lysates were treated by Protein A/G PLUS-Agarose (Santa Cruz Biotechnology, USA) for $1 \mathrm{~h}$. After that, each samples were incubated with the IgG (Proteintech, USA) overnight at $4{ }^{\circ} \mathrm{C}$. Then, the nuclear pellet was collected by centrifugation at $3000 \mathrm{rpm}$ for $5 \mathrm{~min}$ at $4{ }^{\circ} \mathrm{C}$ and subsequently washed by Protein A/G Plus-Agarose beads for four times. The purified proteins were separated by 4-20\% gradient SDS-PAGE. Anti-TRIM27 antibody (12205-1-AP, Proteintech, USA), Anti-PTEN (ab32199, Abcam, UK) and anti-Ubiquitin antibody (ab7780, Abcam, UK) antibody were used for immunoblotting.

\section{Statistical analysis}

Statistical analyses were performed by using the software of GraphPad Prism Version 7.0 (CA, USA). Data were presented by mean $\pm S D$. ANOVA for multiple comparisons was used to determine statistical significance and $p$-value $<0.05$ was accepted.

\section{Results}

\section{TRIM27 was upregulated in ESCC tissues and cells}

A total of 25 ESCC tissues and 18 adjacent-matched noncancerous samples were used to examine the mRNA level of TRIM27. Moreover, six pairs of ESCC tissues and matched noncancerous samples were utilized to quantify the protein content of TRIM27. Both the mRNA and protein level of TRIM27 were significantly increased in ESCC tissues compared with that of adjacent samples (Fig. 1a, b). Therefore, our results demonstrated that TRIM27 was upregulated in ESCC tissues.

Moreover, qRT-PCR and western blot were used to determine the mRNA and protein level of TRIM27 in four ESCC cell lines, including TE-1, TE-11, EAC-109 and KYSE150. The esophageal epithelial cells HECC were used as control. In the present study, the level of TRIM27 was also upregulated in ESCC cells compared with that of HECC, especially in TE-11 and KYSE150 cells (Fig. 1c, d). Therefore, knockdown of TRIM27 was induced in TE-11 and KYSE150 cells respectively. Meanwhile, TE-1 cells were chosen for overexpression of TRIM27.

\section{Knockdown and overexpression of TRIM27 in ESCC cells}

To further examine the function of TRIM27 in ESCC cells, we synthesized three short interference RNAs (siRNAs) targeting human TRIM27 (siTRIM27-1, siTRIM27-2 and siTRIM27-3) and a nonspecific scrambled siRNA (siNC) and subsequently transfected into KYSE150 and TR-11 cells. The unprocessed cells were served as blank control (BLANK). All TRIM27-siRNAs significantly suppressed the expression of endogenous TRIM27. Cells transfected with siTRIM27-1 and siTRIM27-2 showed a stronger effect than that of siTRIM27-3 in ESCC cells. Therefore, siTRIM27-1 and siTRIM27-2 transfected cells were used for the following analyses (Fig. 2a, b).

Meanwhile, TE-1 cells were transfected with a plasmid for overexpressing TRIM27 (oeTRIM27). Both the mRNA and protein content of TRIM27 was significantly overexpressed in oeTRIM27-transfected cells (Fig. 2c, d). Therefore, the oeTRIM27 transfected cells were utilized for the following analyses.

\section{Knockdown of TRIM27 suppressed the growth of ESCC cells} Cell proliferation rate was examined by cell counting kit-8 (CCK-8). As shown in Fig. 3a, b, siTRIM27-1 and siTRIM27-2 were deeply suppressed the proliferation rate of ESCC cells in two cell lines. Therefore, TRIM27 presented the pro-proliferation property in ESCC cells. Moreover, we also analyzed the apoptosis profile of siTRIM27 transfected cells. Obviously, the apoptosis profile of siTRIM27-1 or siTRIM27-2 transfected cells was 


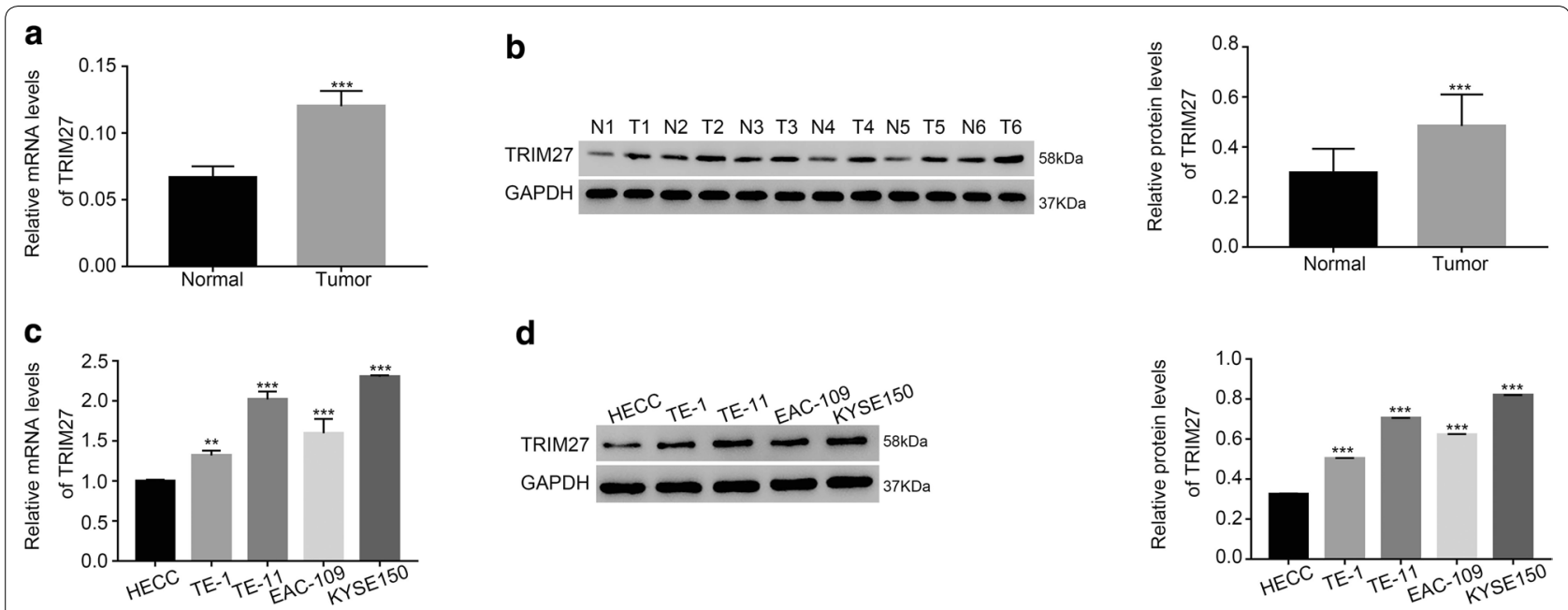

Fig. 1 TRIM27 was upregulated in ESCC tissues and cells. $N$ normal, $T$ tumor. a The relative mRNA level of TRIM27 detected in ESCC tissues ( $n=25)$ and adjacent noncancerous samples $(n=18),{ }^{* * *} p<0.001$ vs normal. $\mathbf{b}$ The protein content of TRIM27 examined in six pairs of ESCC tissues and matched adjacent samples, ${ }^{* * *} p<0.001$ vs normal. $\mathbf{c}$, $\mathbf{d}$ Stands for the mRNA and protein level of TRIM27 in HECC, TE-1, TE-11, EAC-109 and KYSE150 cells respectively, ${ }^{* * *} p<0.001$ vs HECC

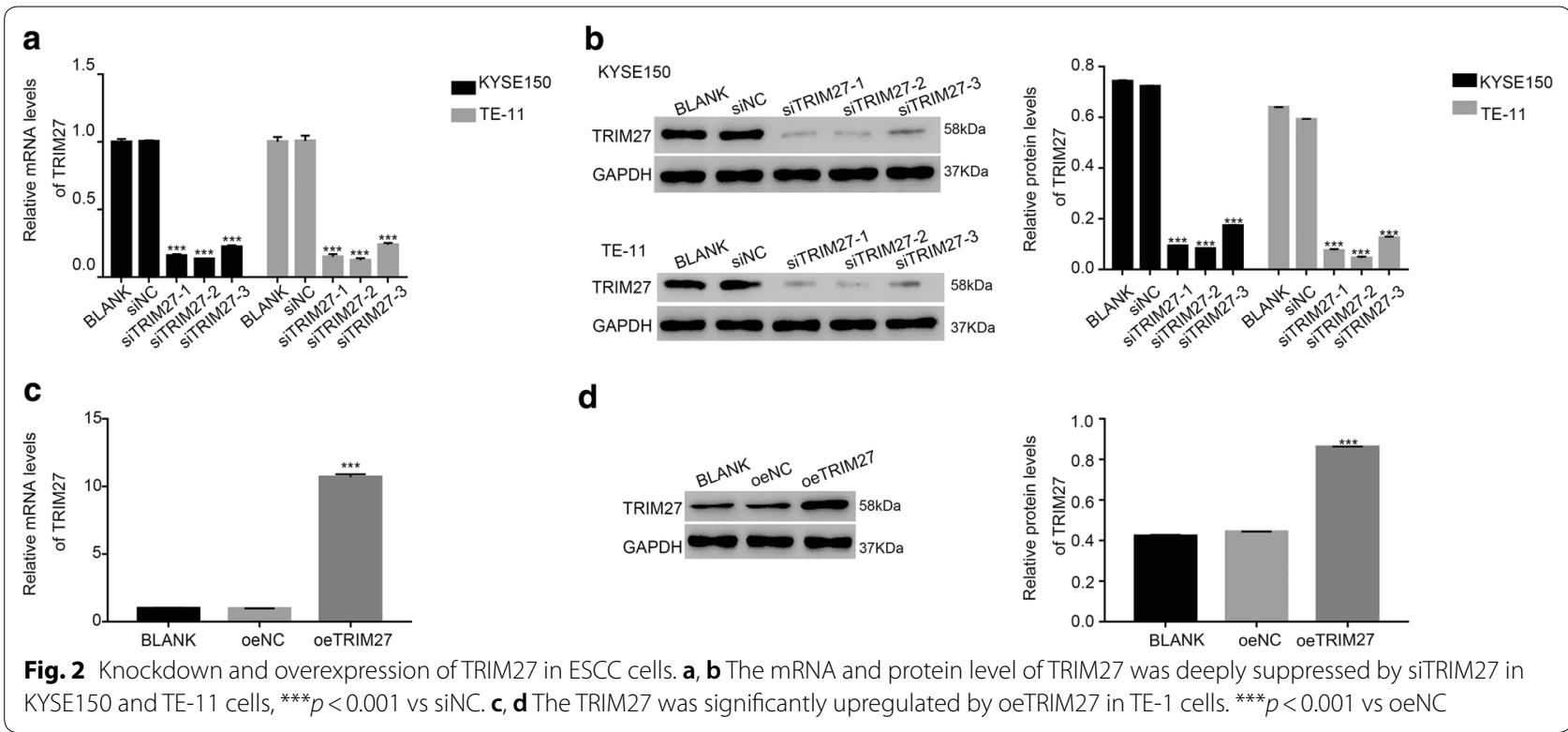

much higher than that of siNC transfected cells (Fig. 3c). These results elucidated the anti-apoptosis function of TRIM27 in ESCC cells.

\section{Glucose transport activity was deeply suppressed by siTRIM27 in ESCC cells}

The abnormal of energy metabolism is one of the biochemical characters for cancer cells, which leads to the acceleration of glycolysis activity [15]. In this study, we analyzed the activity of glycolysis by using the glucose analog 2-NBDG. Interestingly, the level of 2-NBDG was deeply suppressed in ESCC cells transfected with siTRIM27-1 or siTRIM27-2 (Fig. 3d). Therefore, TRIM27 might promote the proliferation of human ESCC cells through enhancing the glycolysis activity.

Previous reports have demonstrated that GLUT1 and HKII are positive correlative with the activity of glycolysis [16-19]. To further examine the function of TRIM27 in glycolysis, we quantified the protein content of GLUT1 and HKII in different cells as indicated. The protein 


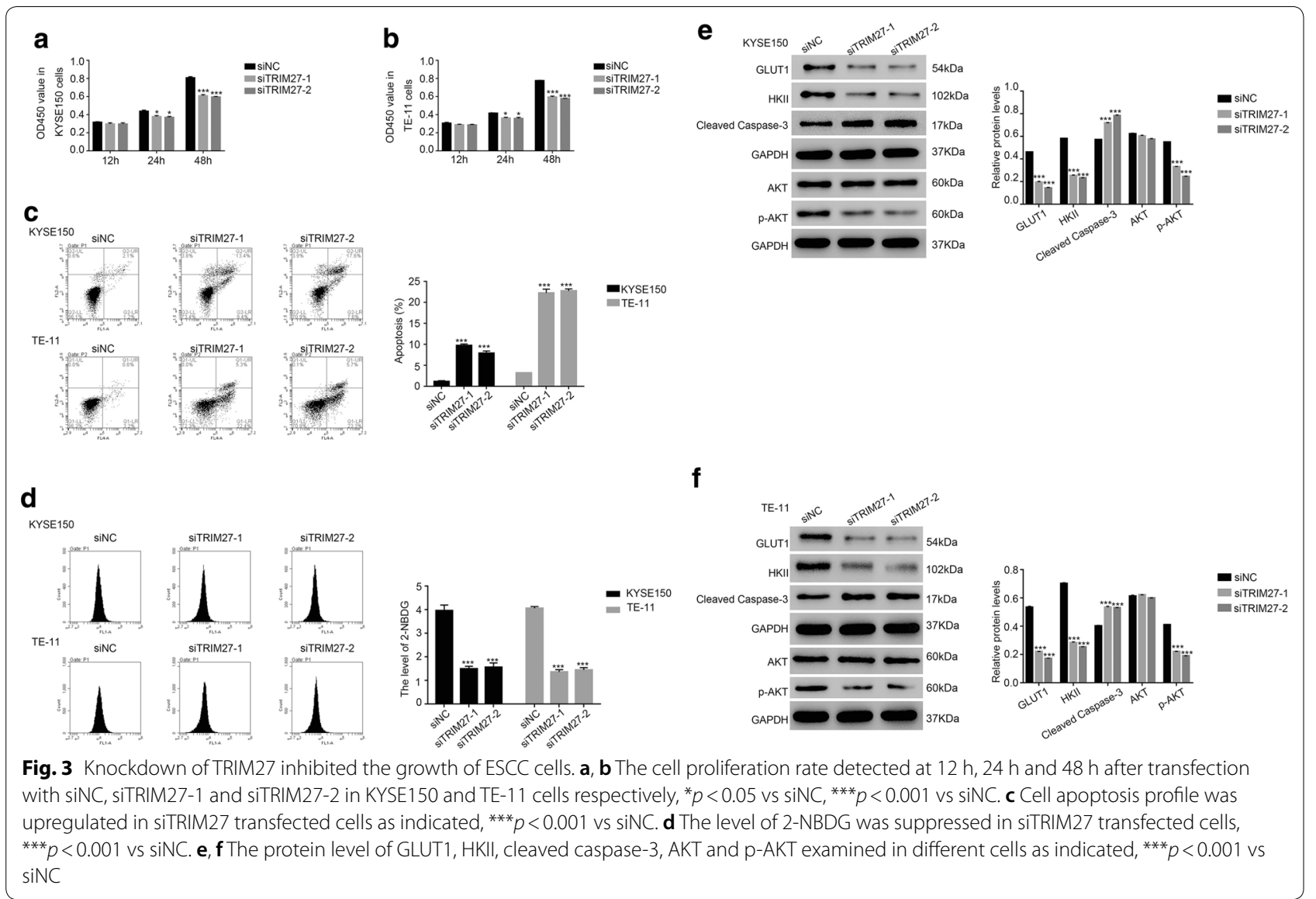

content of GLUT1 and HKII were significantly decreased in siTRIM27 transfected cells (Fig. 3e, f). Moreover, the protein level of cleaved caspased-3 was remarkably upregulated in siTRIM27-1 or siTRIM27-2 transfected cells. In addition, the phosphorylation of AKT (p-AKT) was also inhibited in siTRIM27-1 or siTRIM27-2 transfected cells.

The AKT inhibitor LY29400 and glycolytic inhibitor 3-BrPA abolished the function of TRIM27 in ESCC cells

In order to further explore the correlation between TRIM27 and AKT, the AKT inhibitor LY294002 (25 $\mu \mathrm{mol} / \mathrm{L})$ was used to culture cells transfected with oeTRIM27. Meanwhile, the oeTRIM27 transfected cells were also cultured in the present of the glycolytic inhibitor 3-BrPA $(20 \mu \mathrm{mol} / \mathrm{L})$.

The cell proliferation rate was significantly promoted in oeTRIM27 transfected cells (Fig. 4a). However, the AKT inhibitor LY294002 deeply suppressed the cell proliferation rate of oeNC and oeTRIM27 transfected cells. Meanwhile, the similar result was also obtained in 3-BrPA cultured cells. Moreover, the apoptosis rate was deeply inhibited in oeTRIM27 transfected cells.
Interestingly, the inhibitor LY294002 and 3-BrPA remarkably promoted the cell apoptosis rate in oeNC or oeTRIM27 transfected cells (Fig. 4b).

In addition, we also analyzed the activity of glucose transport in different cells as indicated. It was clearly identified that overexpression of TRIM27 promoted the transport of glucose in cells transfected with oeTRIM27. Moreover, the inhibitor LY294002 and 3-BrPA deeply inhibited the glucose transportation in oeTRIM27 transfected cells (Fig. 4c). As shown in Fig. 4d, the protein content of GLUT1 and HKII were significantly increased in oeTRIM27 transfected cells. However, the AKT inhibitor LY294002 deeply inhibited the expression of GLUT1 and HKII in oeNC or oeTRIM27 transfected cells. Moreover, the protein level of cleaved caspase- 3 showed the opposite results as that of GLUT1 and HKII in oeTRIM27 transfected cells. Importantly, the phosphorylation of AKT was positively correlated with TRIM27, which was deeply suppressed by the inhibitor LY294002 and 3-BrPA. Taken together, all these results demonstrated that the effect of TRIM27 was deeply abolished by the AKT inhibitor LY294002 and 3-BrPA on ESCC cells. 


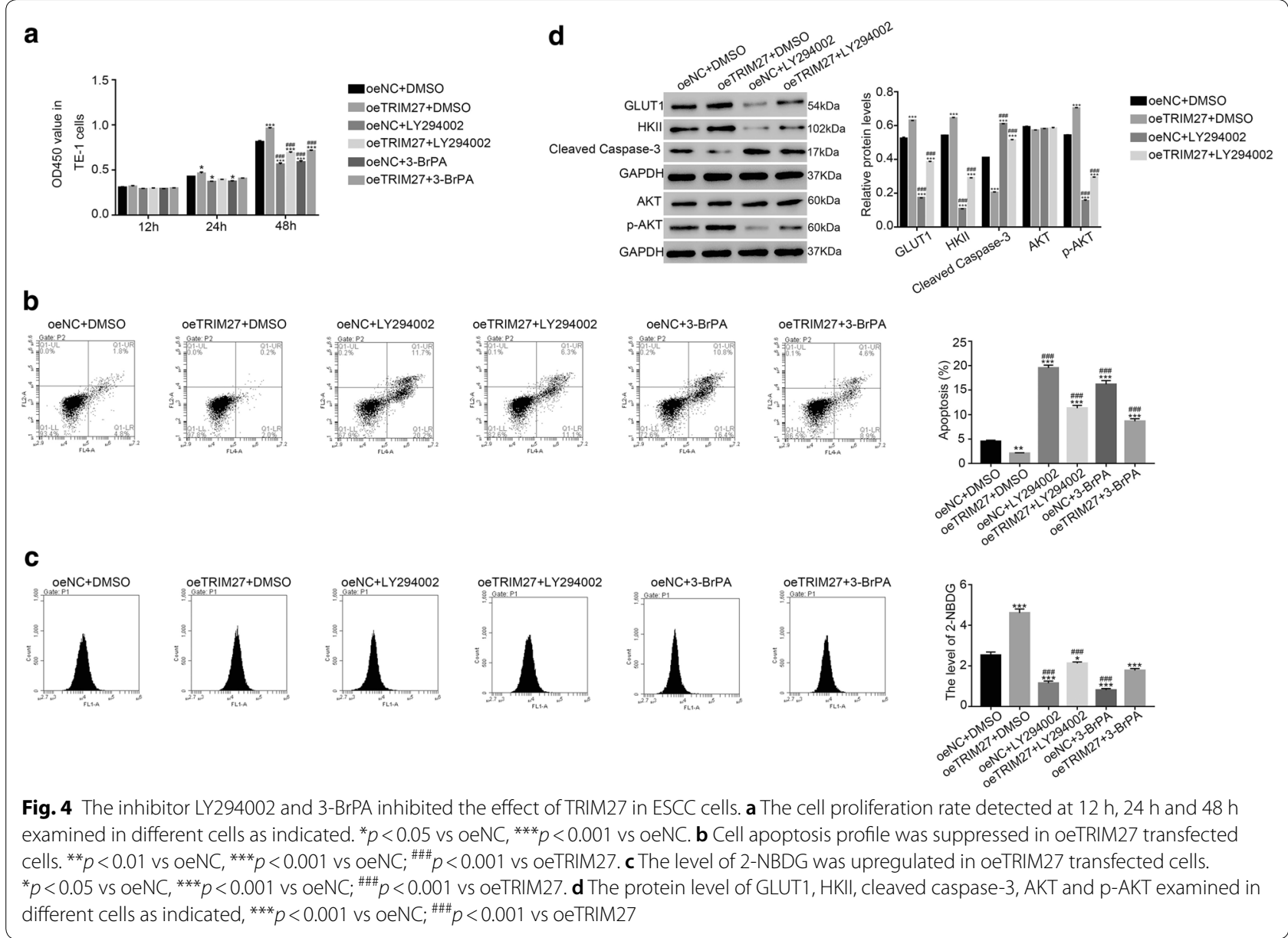

\section{TRIM27 interacted with PTEN and promoted its} poly-ubiquitination in ESCC cells

PTEN is identified as a critical component in PI3/AKT pathway [20]. PTEN/AKT pathway is the common abnormal signaling pathway in human cancers [21]. In order to further examine the function of TRIM27 in PI3/AKT, we analyzed the interaction between TRIM27 and PTEN by co-immunoprecipitation (Co-IP). As shown in Fig. 5a, it was easily identified that there was a stronger interaction between TRIM27 and PTEN. These results indicated that TRIM27 directly interacted with PTEN in ESCC cells.

Previous report has demonstrated that the ubiquitination of PTEN is critical for its stability and nuclear localization [22]. Enhancing the ubiquitination of PTEN has improved the activity of PI3/AKT pathway [23]. In this study, intracellular ubiquitination assays was established to examine whether TRIM27 affected the poly-ubiquitination of PTEN. As shown in Fig. 5b, knockdown of TRIM27 deeply reduced the level of PTEN poly-ubiquitination in ESCC cells. Therefore,

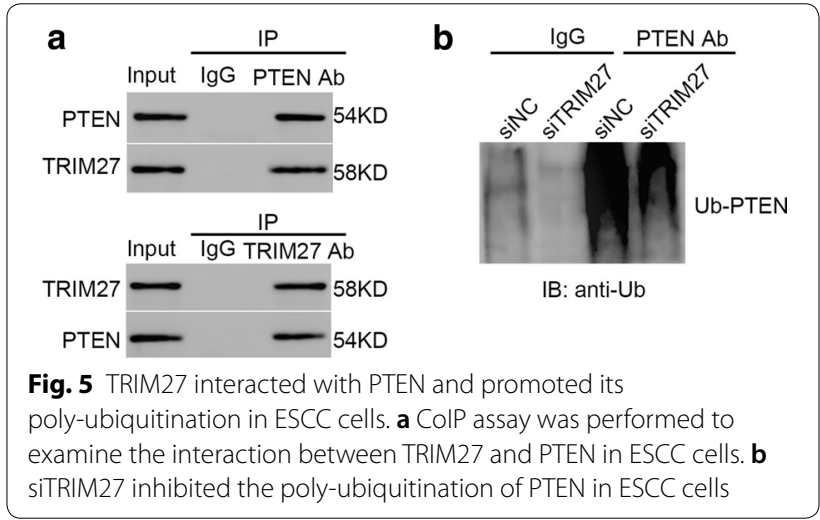

TRIM27 not only interacted with PTEN but also promoted its poly-ubiquitination in ESCC cells.

\section{Discussion}

Esophageal squamous cell carcinoma is one of the common tumors of digestive tract. Numerous deaths are caused by ESCC all over the world [24]. Worse still, the 
outcome of traditional therapy is far from satisfactory. Therefore, enhancing the understanding into the molecule pathogeny of ESCC is a critical step for developing novel strategies for its treatment.

At present study, we systemically explored the function of TRIM27 in ESCC cells. Knockdown and overexpression of TRIM27 were induced in ESCC cells respectively. Analyses from those two experimental sections were consistent. Therefore, our conclusions were more credible.

TRIM protein family are closely related to the development of human tumors [25]. In this study, we found TRIM27 was upregulated in ESCC tissue and cells. Moreover, our results indicated that TRIM27 was a pro-proliferation factor in ESCC cells. Meanwhile, overexpression of TRIM27 deeply inhibited the apoptosis of ESCC cells. Taken together, all these results demonstrated that TRIM27 was a positive oncogene for ESCC.

Growing evidences have indicated that the dysfunction of PI3/AKT signaling pathway is one of the hallmarks for human cancers $[26,27]$. In the present study, our results demonstrated that the AKT inhibitor LY294002 disrupted the function of TRIM27 in ESCC cells. These results illuminated that TRIM27 was involved in PI3/ AKT signaling pathway.

Previous report has demonstrated that PTEN accelerates cell apoptosis via PI3/AKT pathway [28]. Moreover, TRIM27 has enhanced the phosphorylation of AKT pathway in colorectal cancer [5]. In the present research, TRIM27 was identified to interact with PTEN and promoted its poly-ubiquitination. Therefore, our results further confirmed that TRIM27 involved in the p-AKT pathway. Moreover, TRIM27 might inhibit the apoptosis of ESCC cells via enhancing the ubiquitination of PTEN, which subsequently promoted the activity of PI3/AKT signaling pathway in ESCC cells.

Previous report has demonstrated that the glycolysis modulation is the potential anti-cancer therapy [29]. Moreover, some evidences have demonstrated that PTEN/AKT signaling enhances glycolysis in refractory acute myeloid leukemia [30]. PTEN/AKT pathway is reported as a target in suppressing glycolysis activity in cancer cells under hypoxia [31]. In this research, overexpression of TRIM27 promotes the activity of glycolysis metabolism in ESCC cells. Moreover, the protein content of GLUT1 and HKII were positively correlated with the expression of TRIM27. Moreover, the glycolysis inhibitor 3-BrPA deeply suppressed the function of TRIM27 in ESCC cells. Therefore, TRIM27 might promote the activity of glycolysis through upregulating GLUT1 and HKII in ESCC cells. It has been demonstrated that the deficiency of PTEN increases the activity of glycolysis [32]. Hence, the TRIM27/PTEN signaling pathway might also involve in the regulation of glycolysis metabolism in ESCC cells.

\section{Conclusion}

In this study, we induced knockdown and overexpression of TRIM27 in ESCC cells. This research not only gained a deep understanding of TRIM27 but also indicated its possible target and molecule network in ESCC cells.

\section{Supplementary information}

Supplementary information accompanies this paper at https://doi. org/10.1186/s12935-019-0998-4.

Additional file 1. Primer sequence information. Table S1. Human gene TRIM27 (NM_006510.4) RNAi targeting locus information. Table S2. The primary antibodies information.

\section{Abbreviations}

TRIM27: tripartite motif-containing protein 27; ESCC: esophageal squamous cell carcinoma; SNPs: single nucleotide polymorphisms; Co-IP: co-immunoprecipitation; RNAi: RNA interference; cDNA: complementary DNA; CCK-8: cell counting kit-8; IHC: immunohistochemistry; $\mathrm{H} \& \mathrm{E}$ : hematoxylin and eosin; IACUC: Institutional Animal Care and Use Committee.

\section{Acknowledgements}

We acknowledged the support given by The Second Affiliated Hospital of Soochow University, Suzhou, Jiangsu, 224001, China.

\section{Authors' contributions}

The concept of the paper was devised and the first draft was written by ZZ and PC. The experiment was done by LM. NY analyzed the data. All authors read and approved the final manuscript.

\section{Funding}

This work was funded by the Natural and Science Foundation of Nantong (MS12017017-5 and GJZ16075).

\section{Availability of data and materials}

The datasets used and/or analyzed during the current study are available from the corresponding author upon reasonable request.

\section{Ethics approval and consent to participate}

This research was approved by the independent ethics committee of The Second Affiliated Hospital of Soochow University, Suzhou, Jiangsu, China and all patients provided written informed consent, in accordance with the Declaration of Helsinki.

\section{Consent for publication}

Not applicable.

\section{Competing interests}

The authors declare that they have no competing interests.

\footnotetext{
Author details

${ }^{1}$ Department of Oncology, The Second Affiliated Hospital of Soochow University, Sanxiang Road No. 1055, Gusu District, Suzhou 215004, Jiangsu, China. ${ }^{2}$ Department of Oncology, First People's Hospital of Yancheng, The Forth Affiliated Hospital of Nantong University, Yulong West Road No.166, Tinghu District, Yancheng 224001, Jiangsu, China. ${ }^{3}$ Departments of Radiotherapy, Affiliated Hospital of Nantong University, Nantong, Jiangsu, China.
} 
Received: 11 July 2019 Accepted: 23 October 2019

Published online: 08 November 2019

\section{References}

1. Cristina B, Fabio L, Jacques F, Werner G, Franca L, Paola B, Eva N, Carlo LV. Trends in oesophageal cancer incidence and mortality in Europe. Int $J$ Cancer. 2010;122(5):1118-29.

2. Siegel RL, Fedewa SA, Miller KD, Ann GS, Pinheiro PS, Dinorah MT, Ahmedin J. Cancer statistics for Hispanics/Latinos, 2015. CA Cancer J Clin. 2016;65(6):457-80.

3. di Pietro M, Canto MI, Fitzgerald RC. Endoscopic management of early adenocarcinoma and squamous cell carcinoma of the esophagus: screening, diagnosis, and therapy. Gastroenterology. 2018;154(2):421-36.

4. Micale L, Chaignat E, Fusco C, Reymond A, Merla G. The tripartite motif: structure and function. Adv Exp Med Biol. 2012;770:11-25.

5. Zhang Y, Feng Y, Ji D, Wang Q, Qian W, Wang S, Zhang Z, Ji B, Zhang C, Sun $Y$, et al. TRIM27 functions as an oncogene by activating epithelialmesenchymal transition and p-AKT in colorectal cancer. Int J Oncol. 2018;53:620-32.

6. Ma Y, Wei Z, Bast RC Jr, Wang Z, Li Y, Gao M, Liu Y, Wang X, Guo C, Zhang $L$, et al. Downregulation of TRIM27 expression inhibits the proliferation of ovarian cancer cells in vitro and in vivo. Lab Invest. 2016:96(1):37-48.

7. Meng YY, Wang X, Huang DD, Jin QZ, Chen WB, Cai ZY. Knockdown of TRIM27 expression regulates cell proliferation, invasion and migration in human nasopharyngeal 5-8F carcinoma cells. Chin J Pathophysiol. 2017:33(01):53-9.

8. Mohammad Mahabub-Uz Z, Teruaki N, Tsuyoshi T, Tomoo O, Wanzhu J, Toshie S, Yasunori T, Shunsuke I. Ubiquitination-deubiquitination by the TRIM27-USP7 complex regulates tumor necrosis factor alpha-induced apoptosis. Mol Cell Biol. 2013;33(24):4971-84

9. Fang-Fang S, Wen-Bin Y, Fu-You Z, Ying P, Xue-Ke Z, Yan J, Xin S, Bei L, Xue-Na H, Sa T. Variations in the MHC region confer risk to esophageal squamous cell carcinoma on the subjects from high-incidence area in northern China. PLoS ONE. 2014;9(3):e90438.

10. Wang $Q$, Chen $X$, Hay N. Akt as a target for cancer therapy: more is not always better (lessons from studies in mice). Br J Cancer 2017;117(2):159-63.

11. Sun Y, Ji B, Feng Y, Zhang Y, Ji D, Zhu C, Wang S, Zhang C, Zhang D, Sun Y. TRIM59 facilitates the proliferation of colorectal cancer and promotes metastasis via the PI3K/AKT pathway. Oncol Rep. 2017;38(1):43-52.

12. $X u G$, Guo Y, Xu D, Wang $Y$, Shen $Y$, Wang F, Lv Y, Song F, Jiang D, Zhang $Y$. TRIM14 regulates cell proliferation and invasion in osteosarcoma via promotion of the AKT signaling pathway. Sci Rep. 2017;7:42411.

13. He D, Zhang S. UNBS5162 inhibits the proliferation of esophageal cancer squamous cells via the PI3K/AKT signaling pathway. Mol Med Rep. 2017;17(1):549-55.

14. Li B, Wen WX, Lam AKY, Yang W, Hu HF, Xin YG, Yan RQ, Saremi N, Tsao SW, He QY. Significance of PI3K/AKT signaling pathway in metastasis of esophageal squamous cell carcinoma and its potential as a target for anti-metastasis therapy. Oncotarget. 2017;8(24):38755-66.

15. Ganapathy-Kanniappan S, Geschwind JFH. Tumor glycolysis as a target for cancer therapy: progress and prospects. Mol Cancer. 2013;12(1):152.

16. Lu YX, Wu QN, Chen DL, Chen LZ, Wang ZX, Ren C, Mo HY, Chen Y, Sheng $\mathrm{H}$, Wang $Y N$, et al. Pharmacological ascorbate suppresses growth of gastric cancer cells with GLUT1 overexpression and enhances the efficacy of oxaliplatin through redox modulation. Theranostics. 2018;8(5):1312-26.

17. Riskin A, Mond Y. Prolactin-induced subcellular targeting of GLUT1 glucose transporter in living mammary epithelial cells. Rambam Maimonides Med J. 2015. https://doi.org/10.5041/RMMJ.10223.

18. Liu Y, Murray-Stewart T, Casero RA Jr, Kagiampakis I, Jin L, Zhang J, Wang $\mathrm{H}$, Che Q, Tong H, Ke J, et al. Targeting hexokinase 2 inhibition promotes radiosensitization in HPV16 E7-induced cervical cancer and suppresses tumor growth. Int J Oncol. 2017;50(6):2011-23.

19. Sun $X$, Zhang L. MicroRNA-143 suppresses oral squamous cell carcinoma cell growth, invasion and glucose metabolism through targeting hexokinase 2. Biosci Rep. 2017. https://doi.org/10.1042/BSR20160404.

20. Joshi A, Ellenson LH. PI3K/PTEN/AKT genetic mouse models of endometrial carcinoma. Adv Exp Med Biol. 2017:943:261-73.

21. Zhang S, Zhang Y, Qu J, Che X, Fan Y, Hou K, Guo T, Deng G, Song N, Li C. Exosomes promote cetuximab resistance via the PTEN/Akt pathway in colon cancer cells. Braz J Med Biol Res. 2018;51(1):e6472.

22. Shao C, Li Z, Ahmad N, Liu X. Regulation of PTEN degradation and NEDD4-1 E3 ligase activity by Numb. Cell Cycle. 2017;16(10):957.

23. Lee JT, Jing S, Jiayun Z, Muyang L, Brenda Z, Amanda Z, Ramon P, Wei G. RFP-mediated ubiquitination of PTEN modulates its effect on AKT activation. Cell Res. 2013;23(4):552-64.

24. Chen W, Zheng R, Zhang S, Zhao P, Zeng H, Zou X, He J. Annual report on status of cancer in China, 2010. Chin J Cancer Res. 2014;26(1):48-58.

25. Hatakeyama S. TRIM family proteins: roles in autophagy, immunity, and carcinogenesis. Trends Biochem Sci. 2017;42(4):297.

26. Brown JS, Banerji U. Maximising the potential of AKT inhibitors as anticancer treatments. Pharmacol Ther. 2016;172:101.

27. Miriam M, Maria Chiara DS, Laura B, Federico G, Emilio H. PI3K AKT signaling pathway and cancer: an updated review. Ann Med. 2014:46(6):372-83.

28. Jia M, Chen X, Liu J, Chen J. PTEN promotes apoptosis of $\mathrm{H}_{2} \mathrm{O}_{2}$-injured rat nasal epithelial cells through PI3K/Akt and other pathways. Mol Med Rep. 2017; 17(1):571.

29. Gill KS, Fernandes P, Donovan TR, Mckenna SL, Doddakula KK, Power DG, Soden DM, Forde PF. Glycolysis inhibition as a cancer treatment and its role in an anti-tumour immune response. Biochim Biophys Acta. 2016:1866(1):87-105.

30. Ryu MJ, Han J, Kim SJ, Lee MJ, Ju X, Lee YL, Son JH, Cui J, Jang Y, Chung W, Song IC, Kweon GR, Heo JY. PTEN/AKT signaling mediates chemoresistance in refractory acute myeloid leukemia through enhanced glycolysis. Oncol Rep. 2019:42(5):2149-58.

31. Fenglin C, Mingkai Z, Canmei Z, Jun P, Xiaozhong W, Jianying L, Zhixin C, Yuehong $\mathrm{H}$. Baicalein reverses hypoxia-induced 5-FU resistance in gastric cancer AGS cells through suppression of glycolysis and the PTEN/Akt/ HIF-1a signaling pathway. Oncol Rep. 2015;33(1):457-63.

32. Blouin MJ, Zhao Y, Zakikhani M, Algire C, Piura E, Pollak M. Loss of function of PTEN alters the relationship between glucose concentration and cell proliferation, increases glycolysis, and sensitizes cells to 2-deoxyglucose. Cancer Lett. 2010;289(2):246-53.

\section{Publisher's Note}

Springer Nature remains neutral with regard to jurisdictional claims in published maps and institutional affiliations. 